Proceedings of the Edinburgh Mathematical Society (2002) 45, 507-511 (C)

DOI:10.1017/S0013091501000050 Printed in the United Kingdom

\title{
PRIME RINGS WITH FINITENESS PROPERTIES ON ONE-SIDED IDEALS
}

\author{
TSIU-KWEN LEE \\ Department of Mathematics, National Taiwan University, \\ Taipei 106, Taiwan (tklee@math.ntu.edu.tw)
}

(Received 15 January 2001)

\begin{abstract}
Let $R$ be a prime ring with extended centroid $C, \rho$ a non-zero right ideal of $R$ and let $f\left(X_{1}, \ldots, X_{t}\right)$ be a polynomial, having no constant term, over $C$. Suppose that $f\left(X_{1}, \ldots, X_{t}\right)$ is not central-valued on $R C$. We denote by $f(\rho)$ the additive subgroup of $R C$ generated by all elements $f\left(x_{1}, \ldots, x_{t}\right)$ for $x_{i} \in \rho$. The main goals of this note are to prove two results concerning the extension properties of finiteness conditions as follows.
\end{abstract}

(I) If $f(\rho)$ spans a non-zero finite-dimensional $C$-subspace of $R C$, then $\operatorname{dim}_{C} R C$ is finite.

(II) If $f(\rho) \neq 0$ and is a finite set, then $R$ itself is a finite ring.

Keywords: prime ring; polynomial identity (PI); generalized polynomial identity (GPI); extended centroid

AMS 2000 Mathematics subject classification: Primary 16N60; 16R50

\section{Introduction and results}

Throughout this note, $R$ always denotes a prime ring with extended centroid $C$ and $\rho$ a non-zero right ideal of $R$. The goal of this note is to study the extension properties of certain finiteness conditions from one-sided ideals of a prime ring to the whole prime ring. We were motivated by two elementary observations: (I) if $\operatorname{dim}_{C} \rho C$ is finite, then so is $\operatorname{dim}_{C} R C$; and (II) if $\rho$ is a finite subset of $R$, then so is $R$. For (I) see [3, Lemma 1]. For (II), since, by the primeness of $R, R$ can be embedded in $\operatorname{End}(\rho,+, 0)$ via right multiplications and $\operatorname{End}(\rho,+, 0)$ is a finite set, $R$ itself is a finite ring. In a recent paper Bell proved the following theorem.

Theorem. Suppose that $\rho$ is of finite index in $R$ and $[\rho, \rho]$ is finite. Then $R$ is either finite or commutative (see [1, Theorem 2.2]).

Since, in Bell's Theorem, $[\rho, \rho]$ contains all elements $x y-y x$ for all $x, y \in \rho$, we want to extend these results above to more generalized forms. In particular, we shall see that, in Bell's Theorem, the assumption that $\rho$ is of finite index in $R$ is superfluous. Our point of view in this note is different from that of $[\mathbf{1}]$. To state our results we require some notation. For a polynomial $f\left(X_{1}, \ldots, X_{t}\right)$ over $C$, where the $X_{i}$ are non-commuting indeterminates, 
we denote by $f(\rho)$ the additive subgroup of $R C$ generated by all elements $f\left(x_{1}, \ldots, x_{t}\right)$ for $x_{i} \in \rho$. For $x, y \in R$, set $[x, y]_{0}=x,[x, y]_{1}=[x, y]=x y-y x$ and $[x, y]_{k}=\left[[x, y]_{k-1}, y\right]$ for $k>1$. Also, $[\rho, \rho]_{k}$ denotes the additive subgroup of $R$ generated by all elements $[x, y]_{k}$ for $x, y \in \rho$. We are now ready to state our results.

Theorem 1.1. Let $R$ be a prime ring with extended centroid $C, \rho$ a non-zero right ideal of $R$ and $f\left(X_{1}, \ldots, X_{t}\right)$ a polynomial, having no constant term, over $C$. Suppose that $f\left(X_{1}, \ldots, X_{t}\right)$ is not central-valued on $R C$.

(I) Suppose that $f(\rho)$ spans a non-zero finite-dimensional $C$-subspace of $R C$. Then $\operatorname{dim}_{C} R C$ is finite.

(II) Suppose that $f(\rho) \neq 0$ and is a finite set. Then $R$ itself is a finite ring.

Corollary 1.2. Let $R$ be a prime ring with extended centroid $C, \rho$ a non-zero right ideal of $R$, and $k$ a non-negative integer.

(I) Suppose that $[\rho, \rho]_{k}$ spans a finite-dimensional $C$-subspace of $R C$. Then $\operatorname{dim}_{C} R C$ is finite.

(II) Suppose that $[\rho, \rho]_{k}$ is a finite set. Then $R$ is either commutative or finite.

Proof. In view of Theorem 1.1, it suffices to prove that $[\rho, \rho]_{k} \not \subset C$ unless $R$ is commutative. Indeed, suppose that $[\rho, \rho]_{k} \subseteq C$. Then $[x, y]_{k+1}=0$ for all $x, y \in \rho$. In view of [5, Lemma 1], $R$ is commutative. This proves the corollary.

We remark that Corollary 1.2 (II) gives a generalization of Bell's Theorem [1, Theorem 2.2].

\section{Proof of Theorem 1.1}

To prove Theorem 1.1 we need the following two theorems $[\mathbf{2}$, Theorem 1 (II) (i) and Theorem 2]. Recall that a prime ring $R$ is called centrally closed if $R=R C$ and that a right ideal of a ring is called a polynomial identity (PI) right ideal if the right ideal is itself a PI-ring. We write $\mathrm{M}_{2}(\mathrm{GF}(2))$ to stand for the 2 by 2 matrix ring over $\mathrm{GF}(2)$, the Galois field of two elements.

Theorem 2.1. Let $R$ be a centrally closed prime $C$-algebra and $f\left(X_{1}, \ldots, X_{t}\right)$ a nonzero polynomial over $C$. Suppose that $\rho$ is a PI right ideal of $R$ such that $f(\rho) \neq 0$. Then there exists an idempotent $e$ in the socle of $R$ such that $\rho=e R$ and $e R(1-e) \subseteq f(\rho)$.

Theorem 2.2. Let $R$ be a prime ring with extended centroid $C$ and $I$ a non-zero ideal of $R$. Suppose that $f\left(X_{1}, \ldots, X_{t}\right)$ is a polynomial over $C$ which is not central-valued on $R C$. Then $[M, R] \subseteq f(I)$ for some non-zero ideal $M$ of $R$, except when $R \cong \mathrm{M}_{2}(\mathrm{GF}(2))$ and $f(R)=\left\{0, e_{12}+e_{21}, 1+e_{12}, 1+e_{21}\right\}$ or $\left\{0,1, e_{11}+e_{12}+e_{21}, e_{22}+e_{12}+e_{21}\right\}$. 
From now on, $R$ is always a prime ring with extended centroid $C, \rho$ a non-zero right ideal and $f\left(X_{1}, \ldots, X_{t}\right)$ a non-zero polynomial, having no constant term, over $C$. We denote by $d_{n}\left(X_{1}, \ldots, X_{n} ; Y_{1}, \ldots, Y_{n-1}\right)$ the Capelli polynomial of degree $2 n-1$, that is

$$
d_{n}\left(X_{1}, \ldots, X_{n} ; Y_{1}, \ldots, Y_{n-1}\right)=\sum_{\sigma \in S_{n}}(-1)^{\sigma} X_{\sigma(1)} Y_{1} X_{\sigma(2)} \cdots Y_{n-1} X_{\sigma(n)}
$$

where $X_{i}$ and $Y_{j}$ are non-commuting indeterminates. To apply Theorem 2.1 to our case, we require the following lemma.

Lemma 2.3. Suppose that $f(\rho) \neq 0$. Then

(I) if the additive subgroup $f(\rho)$ spans a finite-dimensional $C$-space, then $f(\rho) C=$ $f(\rho C) C ;$ and

(II) if $f(\rho)$ is finite, then so are both $C$ and $f(\rho C)$.

Proof. (I) Suppose that $\operatorname{dim}_{C} f(\rho) C=n<\infty$. Then

$$
d_{n+1}\left(f\left(x_{11}, \ldots, x_{1 t}\right), \ldots, f\left(x_{n+11}, \ldots, x_{n+1 t}\right) ; y_{1}, \ldots, y_{n}\right)=0
$$

for all $x_{i j} \in \rho$ and all $y_{i} \in R$. Since $\rho$ and $\rho C$ satisfy the same generalized polynomial identities (GPIs) [4, Lemma 2], equation (2.1) still holds for all $x_{i j} \in \rho C$ and all $y_{i} \in R C$. In view of [7, Theorem 7.6.16], for $x_{i j} \in \rho C$ the $n+1$ elements $f\left(x_{11}, \ldots, x_{1 t}\right), \ldots$, $f\left(x_{n+11}, \ldots, x_{n+1 t}\right)$ are $C$-dependent and so $\operatorname{dim}_{C} f(\rho C) C \leqslant n$. But since $f(\rho) C \subseteq$ $f(\rho C) C$, we conclude that $f(\rho) C=f(\rho C) C$ as asserted.

(II) Suppose that $f(\rho)$ is a finite set. Write

$$
f\left(X_{1}, \ldots, X_{t}\right)=\sum_{i=1}^{m} f_{i}\left(X_{1}, \ldots, X_{t}\right)
$$

where $f_{i}\left(X_{1}, \ldots, X_{t}\right)$ is the homogeneous part of $f\left(X_{1}, \ldots, X_{t}\right)$ of degree $i$ for $1 \leqslant i \leqslant m$. Suppose on the contrary that $C$ is infinite. Choose $m$ distinct elements $\beta_{1}, \ldots, \beta_{m}$ in $C$ and then a non-zero ideal $I$ of $R$ such that $\beta_{i} I \subseteq R$. Then $\beta_{i} \rho \subseteq \subseteq \rho$ for each $i$. Applying a standard determinant argument to equation $(2.2)$ we see that $f_{i}(\rho I)$ is finite for each $i$. Since $f(\rho) \neq 0$, we see that $f_{j}(\rho) \neq 0$ for some $j$. Thus we may assume from the start that $f\left(X_{1}, \ldots, X_{t}\right)$ is homogeneous of degree $m \geqslant 1$. Suppose that $f(\rho)$ consists of $\ell$ elements. Since $C$ is an infinite field, we can choose $\mu_{1}, \ldots, \mu_{\ell+1}$ in $C$ such that $\mu_{i}^{m} \neq \mu_{j}^{m}$ for $i \neq j$. Let $J$ be a non-zero ideal of $R$ such that $\mu_{i} J \subseteq R$ for each $i$. In view of $\left[4\right.$, Lemma 2], there exist $x_{i} \in \rho J, 1 \leqslant i \leqslant t$, such that $f\left(x_{1}, \ldots, x_{t}\right) \neq 0$. Now, we see that $\mu_{i}^{m} f\left(x_{1}, \ldots, x_{t}\right)=f\left(\mu_{i} x_{1}, \ldots, \mu_{i} x_{t}\right) \in f(\rho)$ for each $i$. This derives a contradiction, as the set $\left\{\mu_{i}^{m} f\left(x_{1}, \ldots, x_{t}\right) \mid 1 \leqslant i \leqslant \ell+1\right\}$ consists of $\ell+1$ elements. Thus $C$ is a finite field. By (I) we see that $f(\rho) C=f(\rho C) C$. But since $f(\rho)$ and $C$ are finite sets, this implies that $f(\rho C)$ is a finite set, proving (II). 
Lemma 2.4. Let $R$ be a centrally closed prime $C$-algebra with non-zero socle $H$. Suppose that $e$ is a non-trivial idempotent in $H$.

(I) If $\operatorname{dim}_{C} e R(1-e)<\infty$, then $R$ is finite dimensional over $C$.

(II) If $e R(1-e)$ is a finite set, then $R$ is a finite ring.

Proof. (I) Suppose that $\operatorname{dim}_{C} e R(1-e)=n<\infty$. Note that $e$ and $1-e$ are $C$ independent. Thus we see that

$$
S_{n+1}\left(e X_{1}(1-e) Y, \ldots, e X_{n+1}(1-e) Y\right)
$$

is a non-trivial GPI for $R$, where $S_{n+1}\left(X_{1}, \ldots, X_{n+1}\right)$ is the standard polynomial of degree $n+1$. Since $R$ is centrally closed, it follows from Martindale's Theorem [6] that $R$ is a strongly primitive ring. By assumption, $e \in H$ and so $\operatorname{dim}_{C} e R e<\infty$. Thus $\operatorname{dim}_{C} e R=\operatorname{dim}_{C} e R e+\operatorname{dim}_{C} e R(1-e)<\infty$. That is, $R$ contains a non-zero right ideal $e R$, which is finite dimensional over $C$. In view of $[\mathbf{3}$, Lemma 1$], \operatorname{dim}_{C} R<\infty$ follows. This proves (I).

(II) Since $e R(1-e) \neq 0$, we choose $x_{0} \in R$ such that $e x_{0}(1-e) \neq 0$. But $C e x_{0}(1-e) \subseteq$ $e R(1-e)$, so the finiteness of $e R(1-e)$ implies that $C$ is a finite field. By (I), $R$ is finite dimensional over $C$ and so $R$ is a finite ring, proving (II).

We are now in a position to prove Theorem 1.1.

Proof of Theorem 1.1. (I) Suppose that $f(\rho)$ spans a non-zero finite-dimensional $C$-subspace of $R C$. It follows from Lemma 2.3 (I) that $0 \neq f(\rho C)$ is finite dimensional over $C$. Clearly, $\rho C$ is a PI right ideal of $R C$. Note that $R C$ is a centrally closed prime $C$-algebra. In view of Theorem 2.1, there exists an idempotent $e$ in the socle of $R C$ such that $\rho C=e R C$ and $e R C(1-e) \subseteq f(\rho C)$ as $f(\rho C) \neq 0$. If $e \neq 1$, applying Lemma 2.4 we conclude that $\operatorname{dim}_{C} R C$ is finite. Suppose next that $e=1$. Then $\rho C=R C$. Since $f\left(X_{1}, \ldots, X_{t}\right)$ is not central-valued on $R C$, by Theorem 2.2 there exists a non-zero ideal $I$ of $R C$ such that $[I, R C] \subseteq f(\rho C)$ unless $R=R C \cong \mathrm{M}_{2}(\mathrm{GF}(2))$. There is nothing to prove for the exceptional case. Therefore, we may assume that $[I, R C] \subseteq f(\rho C)$ and so $\operatorname{dim}_{C}[I, R C]<\infty$, implying that $R$ itself is a PI-ring. Applying Posner's Theorem yields that $\operatorname{dim}_{C} R C$ is finite.

(II) Suppose that $f(\rho) \neq 0$ and is a finite set. By Lemma 2.3, $f(\rho C)$ is a finite set. Clearly, in this case $\rho C$ must be a PI right ideal of $R$. In view of Theorem 2.1, there exists an idempotent $e$ in the socle of $R C$ such that $\rho C=e R C$ and $e R C(1-e) \subseteq f(\rho C)$. If $e \neq 1$, Lemma 2.4 implies that $R$ is a finite ring. Otherwise, $e=1$ follows and so $\rho C=R C$. As in the argument given in (I), we may assume that $R \neq \mathrm{M}_{2}(\mathrm{GF}(2))$. By Theorem 2.2 there exists a non-zero ideal $I$ of $R C$ such that $[I, R C] \subseteq f(\rho C)$ and so $[I, R C]$ is a finite set. But since $R$ is not commutative, $[I, R C] \neq 0$ and so $C$ must be finite. Now, $R C$ is a PI-ring with $C$ a finite field. It is now clear that $R$ itself is a finite ring, proving (II). 


\section{References}

1. H. E. Bell, A note on centralizers, Int. J. Math. Math. Sci. 24 (2000), 55-57.

2. C. M. Chang And T. K. LeE, Additive subgroups generated by polynomial values on right ideals, Commun. Algebra 29 (2001), 2977-2984.

3. C. L. Chunang, GPIs having coefficients in Utumi quotient rings, Proc. Am. Math. Soc. 103 (1988), 723-728.

4. T. K. LEe, Left annihilators characterized by GPIs, Trans. Am. Math. Soc. 347 (1995), 3159-3165.

5. T. K. LEE, Semiprime rings with hypercentral derivations, Can. Math. Bull. 38 (1995), 445-449.

6. W. S. Martindale III, Prime rings satisfying a generalized polynomial identity, J. Algebra 12 (1969), 576-584.

7. L. H. Rowen, Polynomial identities in ring theory (Academic, 1980). 